\title{
Full waveform moment tensor inversion by reciprocal finite difference Green's function
}

\author{
Taro Okamoto \\ Department of Earth and Planetary Sciences, Tokyo Institute of Technology, 2-12-1 Ookayama, Meguro, Tokyo 152-8551, Japan
}

(Received July 18, 2001; Revised April 6, 2002; Accepted April 9, 2002)

\begin{abstract}
We study two important aspects of the waveform moment tensor inversion for the shallow earthquakes in the subduction region: the effect of the intense lateral inhomogeneity in the structure, and the strategy to invert the waveform data for the focal mechanisms. For the first aspect, using a "forward" finite difference modeling, we demonstrate that the effect of the inhomogeneity is quite large on the surface waves with a period of about $20 \mathrm{~s}$, and the current knowledge on the subduction region structure is practically effective in reproducing the characteristics in the observed waveforms. For the second aspect, we develop a "reciprocal" moment tensor inversion method that can generate the Green's functions for a large quantity of source locations (19,200 in this study) in a realistic inhomogeneous structure by only three finite difference calculations per a single station. The inversion with a grid search scheme result in a reasonable source location, moment tensor and fit of the waveforms using data from only two stations. The constraint on the epicenter in the "transverse" direction is found to be somewhat weak in the case of single-station inversions, but the two-station inversion improves the constraint.
\end{abstract}

\section{Introduction}

The waveform inversion of the moment tensor of the earthquakes provides important contributions to the study of tectonics and earthquake source physics. Many projects for routine determination of the moment tensor have been established and being kept for years (e.g., Dziewonski and Woodhouse, 1983; Sipkin, 1994; Kawakatsu, 1995; Pasyanos et al., 1996; Fukuyama et al., 1998). Also, waveform inversion is the basis of the rupture process analysis of the fault (e.g., Kikuchi and Kanamori, 1991). The current routine regional analysis usually assumes a laterally homogeneous structure in calculating the synthetic waveforms (Green's functions) although the actual structure is often strongly laterally inhomogeneous. Such inhomogeneity has non-negligible effects especially on the surface waves such as the changes in the phase velocity (e.g., Thio and Kanamori, 1995) or the curved paths and multiple paths (e.g., Tanimoto, 1990) and can be the cause of the artificial noise in the inversion process. The most straightforward way to remove this problem is to calculate the synthetic waveforms using realistic laterally inhomogeneous structure.

In the moment tensor inversions we need at least five Green's functions (under the commonly applied condition $\operatorname{trace}\left(M_{i j}\right)=0$ ) for each source-station pair. This implies five "forward" simulations with five elementary source types per a single station. However, it is not enough because we usually need to search the optimum source location by the inversion procedure itself rather than to fix it in the inversion. Thus the number of required simulations per a single station would be up to five times as many as the number of

Copy right(C) The Society of Geomagnetism and Earth, Planetary and Space Sciences (SGEPSS); The Seismological Society of Japan; The Volcanological Society of Japan; The Geodetic Society of Japan; The Japanese Society for Planetary Sciences. potential source locations if we performed forward simulations for every potential sources. This behavior makes the inversion very impractical as the simulation of seismic waves in inhomogeneous structure is still quite numerically intensive.

A more practical strategy is the use of "reciprocal" modeling (e.g., Okamoto, 1994a, b): based on the reciprocal theorem, a single force is applied at the station location, and the response strain recorded at the source location is convolved with the moment tensor to generate the seismic displacement at the station location. The strain time functions for a number of arbitrary source locations can be stored in a single simulation if we use methods like finite difference or finite element. Thus a large number of Green's functions can be calculated by only three simulations (for three components) per a single station, which makes the inversion procedure very feasible.

In this short paper we demonstrate the importance of the lateral inhomogeneity in generating the synthetic waveforms, and show the results of the reciprocal moment tensor inversions with a grid search scheme for the optimum source location and focal mechanism.

\section{Data and Forward Modeling}

We select an event in the Japan trench on 23 Feb. 1995 05:01 UTC $\left(M_{W} 6.2\right)$ as a typical example of the shallow event in the subduction region where the lateral inhomogeneity is very strong. The broadband waveform data at two stations (KGJ and AOB: Fig. 1) maintained by Research Center for Prediction of Earthquakes and Volcanic Eruptions, Graduate School of Science, Tohoku University are downloaded using the GOPHER system installed in Earthquake Research Institute, University of Tokyo.

We assume a model structure for the Japan trench based on the results of the seismic refraction experiments (Murauchi 


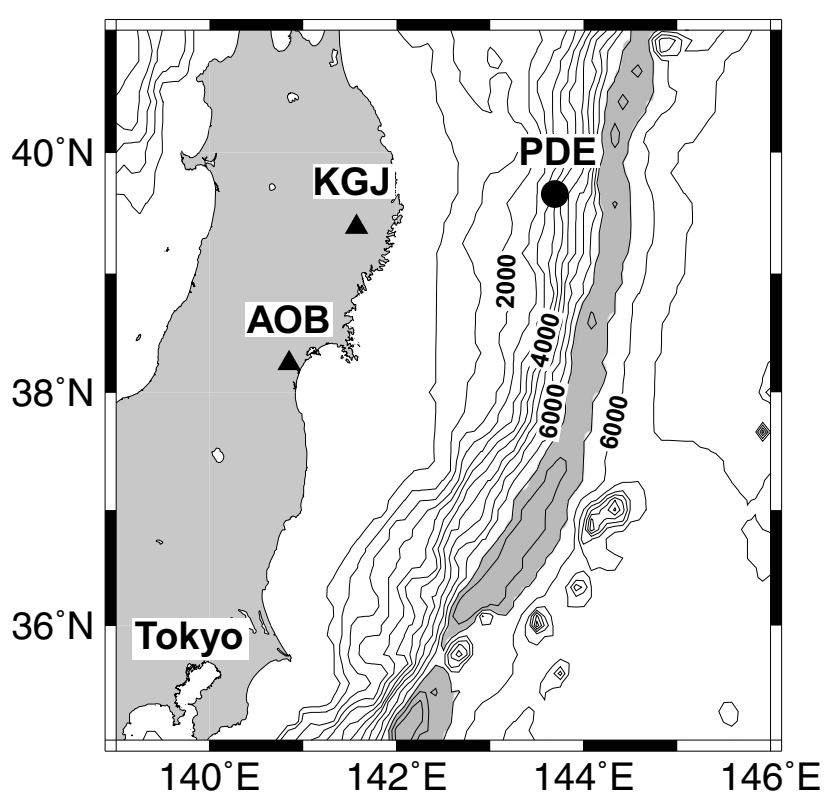

Fig. 1. The PDE epicenter of the event (solid circle) and the stations (solid triangles). The contour interval in the oceanic region is $500 \mathrm{~m}$. Shaded area (deeper than $6500 \mathrm{~m}$ ) in the ocean indicates the trench axis.

and Ludwig, 1980; Suyehiro et al., 1986: see Fig. 2 and Table 1). In order to reduce the required computational resources, we use a "2.5D" finite difference method (the structure is $2 \mathrm{D}$ but the wavefield is full-3D and the spatiowavenumber mixed domain is used for calculation: see e.g., Okamoto 1994b; Furumura and Takenaka, 1996). The material properties are assumed to be identical with respect to the trench-parallel axis because of the nearly 2D topography of the studied area. We also assume homogeneous mantles (separated by the oceanic crust) in order to reduce numerical computations although the modeled surface waves can penetrate into the mantle.

Before going to the inversion analysis, it is important to see the effect of the laterally inhomogeneous structure by employing forward modelings. We apply a point source at the PDE epicenter $(39.66 \mathrm{~N}, 143.69 \mathrm{E})$ with a depth of $15 \mathrm{~km}$, the best double couple mechanism of the Harvard CMT (HCMT) solution (strike $190^{\circ}$, dip $13^{\circ}$, slip $81^{\circ}$ ), a seismic moment of $2.15 \times 10^{18} \mathrm{Nm}$, and a Gaussian moment rate function $f(t)=\exp \left(-\alpha t^{2}\right)$ where $\alpha=0.1$. A 10 $100 \mathrm{~s}$ bandpass filter is applied to both of the synthetic and the observed waveforms, but frequency components around $0.05 \mathrm{~Hz}$ (period of $20 \mathrm{~s}$ ) dominate the waveforms as shown in Fig. 3. Also we employ a simulation for a flat, laterally homogeneous structure. We simply adopt the crustal model under the northern Japan with an addition of a thin surface layer (Table 2).

The synthetics for the realistic $2.5 \mathrm{D}$ structure show remarkable resemblance to the observed data (Fig. 3): the dispersive wave train in the observed surface waves are well reproduced in these synthetics. On the other hand, the synthetics for the assumed flat structure show relatively low dispersive behavior and they do not show good resemblance to the data.

It is often possible to reproduce the effect of inhomoge-

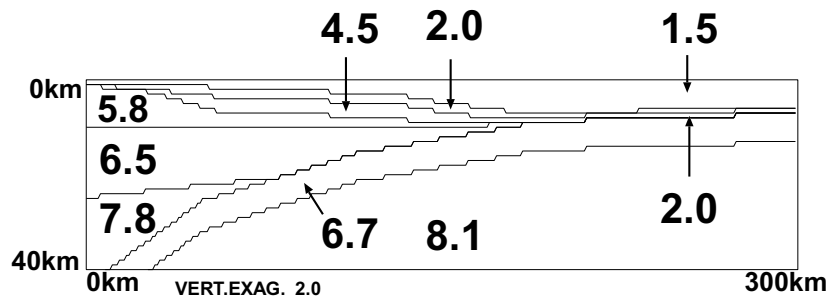

Fig. 2. The vertical cross section of the assumed 2.5D (semi-3D) structure for FDM calculations, which is taken to be perpendicular to the trench axis. The $P$-wave velocities for each layer in $\mathrm{km} / \mathrm{s}$ are indicated. For other material parameters see Table 1. The FDM parameters are: horizontal (trench-normal) and vertical grid spacings of $1 \mathrm{~km}$, minimum wavelength of $5 \mathrm{~km}$ with respect to trench-parallel axis, size of 700 $\mathrm{km}($ trench-normal $) \times 740 \mathrm{~km}$ (trench-parallel) $\times 300 \mathrm{~km}$ (vertical), 121 wavenumbers, time steps of $3000(168 \mathrm{~s})$. The program requires about $212 \mathrm{MB}$ (mega bytes) of memory, and about 34 hours for calculation of one component on Compaq Alpha based machine $(21264 / 666 \mathrm{MHz})$.

Table 1. Structural parameters for the $2.5 \mathrm{D}$ (semi-3D) realistic model. The parameters in a vertical cross section (Fig. 2) are listed. $V_{P}: P$-wave velocity in $\mathrm{km} / \mathrm{s}$. $V_{S}: S$-wave velocity in $\mathrm{km} / \mathrm{s}$. $\rho$ : density in $\mathrm{g} / \mathrm{cm}^{3}$.

\begin{tabular}{llll}
\hline \multicolumn{1}{c}{ layer } & $V_{P}$ & \multicolumn{1}{c}{$V_{S}$} & $\rho$ \\
\hline sea water & 1.5 & 0.0 & 1.0 \\
sediment (1) & 2.0 & 1.0 & 2.0 \\
sediment (2) & 4.5 & 2.6 & 2.5 \\
upper crust & 5.8 & 3.35 & 2.6 \\
lower crust & 6.5 & 3.75 & 2.8 \\
mantle (1) & 7.8 & 4.5 & 3.2 \\
oceanic crust & 6.7 & 3.87 & 2.9 \\
mantle (2) & 8.1 & 4.68 & 3.3 \\
\hline
\end{tabular}

Table 2. Structural parameters for the flat layered model. "d" denotes layer thickness in $\mathrm{km}$.

\begin{tabular}{lllll}
\hline \multicolumn{1}{c}{ layer } & $V_{P}$ & $V_{S}$ & $\rho$ & $\mathrm{d}$ \\
\hline sediment & 4.5 & 2.6 & 2.5 & 2.0 \\
upper crust & 5.8 & 3.35 & 2.6 & 8.0 \\
lower crust & 6.7 & 3.87 & 2.9 & 15.0 \\
mantle & 8.1 & 4.68 & 3.3 & $\infty$ \\
\hline
\end{tabular}

neous structure on the surface waves by adopting a 1D (flatlayered) structure whose phase and group velocities are adjusted to the observational ones averaged over the path, provided the inhomogeneities are not so strong. (In that sense above 1D model is not adjusted for the realistic surface wave velocities.) Detailed study of the limit and/or the applicability of 1D structure is beyond the scope of this short paper, but we here indicate another aspect of the structural effect. Figure 4 shows the synthetic waveforms of a forward modeling with the same parameters as above but for an isotropic (explosive) source and for station AOB. If the structure was a 1D (flat-layered) one there were no transverse component of displacement. But Fig. 4 clearly shows transverse component (i.e., Love wave) with very large amplitude, which is a 

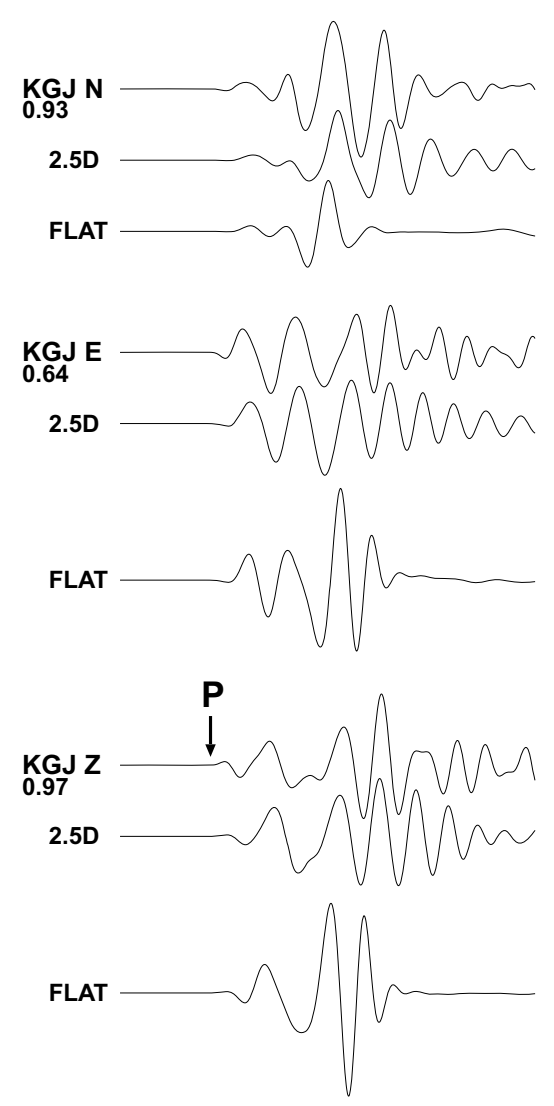

$140.0 s$

Fig. 3. The observed and the "forward" synthetic displacement for station KGJ. "2.5D" denotes the synthetics for the realistic $2.5 \mathrm{D}$ structure, and "FLAT" for the flat layered structure. The number attached to the observed waves are maximum amplitudes in $\mathrm{mm}$.

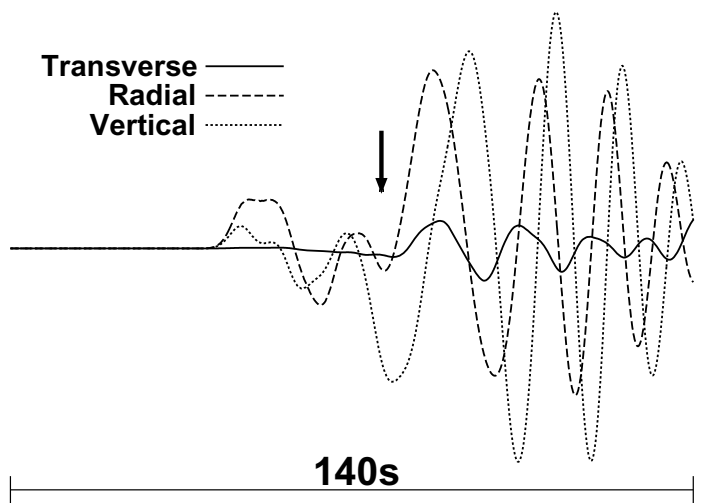

Fig. 4. The synthetic displacement for an isotropic (explosive) source and for station AOB. The FDM region $(900 \mathrm{~km} \times 1140 \mathrm{~km} \times 400 \mathrm{~km})$ is a little larger than that for other calculations. Arrow indicates the onset of surface waves.

result of Rayleigh to Love wave conversion due to the inhomogeneous structure. Such effect cannot be reproduced by any flat-layered model.

These examples show the importance of the laterally inhomogeneous structure in modeling the waveforms, and also show the current knowledge on the structure is practically effective in modeling the waveforms of period around $20 \mathrm{sec}-$ onds. Note that we did not "tune" the structural model in calculating these results but we simply adopted the results of the seismic experiments. However, there still are slight misfits to the data, which may be corrected by searching the optimum source location and the focal mechanism (moment tensor). This is demonstrated in the next section.

\section{Reciprocal Inversion and Results}

Based on the reciprocal theorem, we store the strain components at the grids in a box-shaped region $(195 \mathrm{~km} \times 195$ $\mathrm{km}$ in horizontal and $22 \mathrm{~km}$ in vertical) with $5 \mathrm{~km}$ horizontal and $2 \mathrm{~km}$ vertical spacings. Thus the potential source locations amounts to 19,200 . Our strategy is to perform waveform moment tensor inversion for each of these 19,200 grids and to apply a grid search scheme in order to find the optimum source location. The residual minimized in the waveform inversion is:

$$
S=\frac{1}{3 N_{\text {sta }}} \sum_{i=1}^{N_{\text {sta }}} \sum_{k=1}^{3} \frac{\frac{1}{T} \int_{0}^{T} w(t)^{2}\left[O_{i k}(t)-C_{i k}(t)\right]^{2} d t}{\frac{1}{T} \int_{0}^{T}\left[O_{i k}(t)\right]^{2} d t},
$$

where $N_{\text {sta }}$ denotes the number of stations, $T$ the time duration (110 s from the $P$-wave arrival), $O_{i k}(t)$ and $C_{i k}(t)$ the observed and calculated $k$-th component of the displacement at $i$-th station, respectively, and $w(t)$ the weighting function. We set $w(t)=2$ for the first $25 \mathrm{~s}$ for KGJ and $34 \mathrm{~s}$ for AOB, and $w(t)=1$ for other portion in order to compensate the small amplitude of the body waves. In order to avoid numerical dispersions, we use a smooth Gaussian source function which is slightly broader than the observed first pulse. Because the onset of the synthetic pulse is very emergent (i.e., the amplitude gradually increases), the synthetics are slightly shifted by minimizing the residual $S$. This effectively adjust the overall arrival of the synthetic $P$-wave pulse but its very onset cannot be adjusted to the observation under current numerical parameters. Therefore, we do not discuss the source time (or the centroid time). We employ two single-station inversions (denoted by "KJG" and "AOB" for each station) and one multi(two)-station inversion (denoted by “KGJ+AOB”).
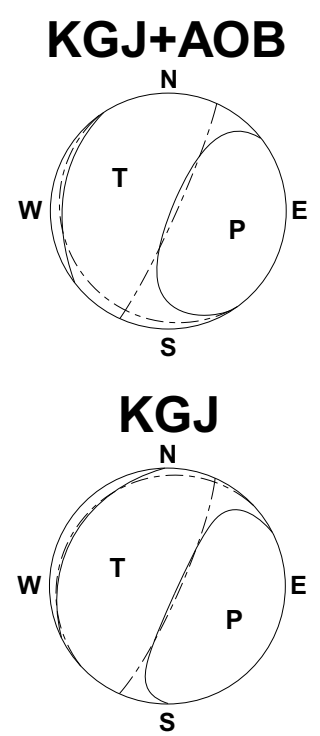
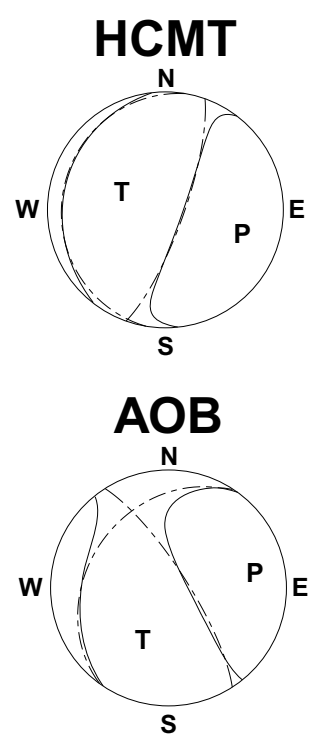

Fig. 5. The moment tensor solutions projected on to the lower hemisphere. The scalar moment of the two-station solution is $1.4 \times 10^{18} \mathrm{Nm}$. 
The HCMT and the moment tensor solutions have similar characteristics except for the single-station solution for AOB (Fig. 5): the principal axes ( $P$ - and $T$-axes) have almost the same directions, and the polarities are nearly the same. Also, the strikes of the steeper nodal planes of the best double couples have nearly NNE-SSW trends consistent with the geometry of the subduction. The strikes of the shallower nodal plane are not constrained well in the present study.

The slightly improper mechanism for AOB (singlestation) is due to the weak constraint on the source location in the "transverse" direction. This is illustrated in Fig. 6 which plots the horizontal cross sections of the residuals sliced through the optimum source depths. The inversion for KGJ (top) shows a distinct, prolonged trough extend-
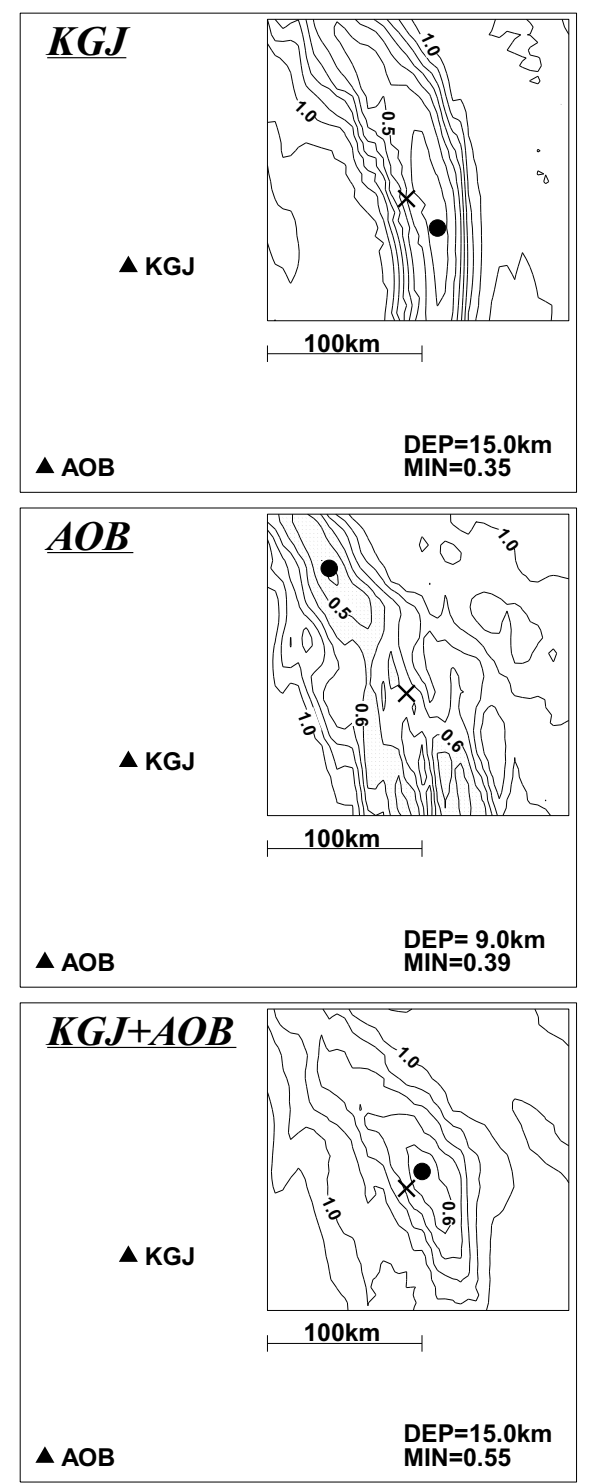

Fig. 6. The horizontal cross sections of the residuals sliced through the optimum source depth. The vertical line of the figure is parallel to the trench axis and the horizontal line is normal to the trench axis. The waveform inversions are done for points in the inner box. The contour interval is 0.1 . Solid circles indicate the optimum points for the source locations. The PDE epicenter (cross) and the two stations (solid triangles) are also projected for reference. The area with residuals smaller than 0.6 are gray-shaded in middle figure to indicate the region of local minima. ing nearly along an arc whose center of curvature is the station KGJ. This indicates a weak constraint on the epicentral parameters in the transverse direction (along the arc). The single-station inversion for AOB (middle) also shows similar feature that the residual trough align along an arc with a center roughly being the station. However, the pattern is more complex and there are many local minima. The solution falls into one of these local minima, which results in a slightly improper mechanism. Also, effects due to the deviation of the actual 3D structure from the assumed 2D model must be larger for AOB, which decrease the accuracy of the synthetics and may be partially responsible for the improper mechanism.

Despite of these uncertainties, the result of the two-station inversion (bottom) shows a single, relatively well defined global minimum. The trough in the residual pattern becomes more compact although it still have some prolonged feature. Note that the best point is not a simple average of the best points of the two single-station inversions, but it occurs approximately at the intersection point of the two arc-like troughs shown in the single-station inversion results. Because both the troughs for KGJ and AOB run through the reasonable point, the two-station inversion yields a reasonable solution (see also Fig. 7).

Figure 7 shows the vertical cross sections of the residual distributions plotted on the assumed structure through the optimum point. The single-station inversion for KGJ (top) results in a fairly well constrained global minimum for the source location. The optimum location is also reasonable because this event is an interplate thrusting one. This shows a proper constraints on the source position in the "radial" and

KGJ

0.35

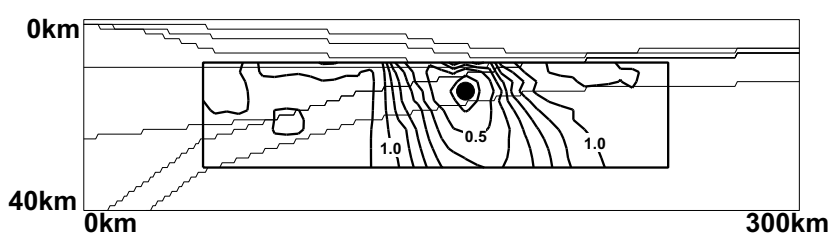

AOB
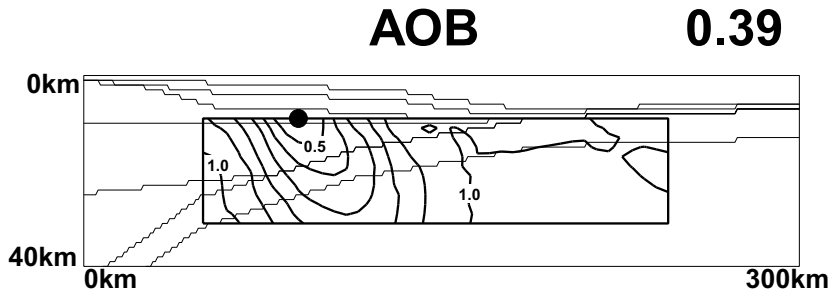

KGJ+AOB
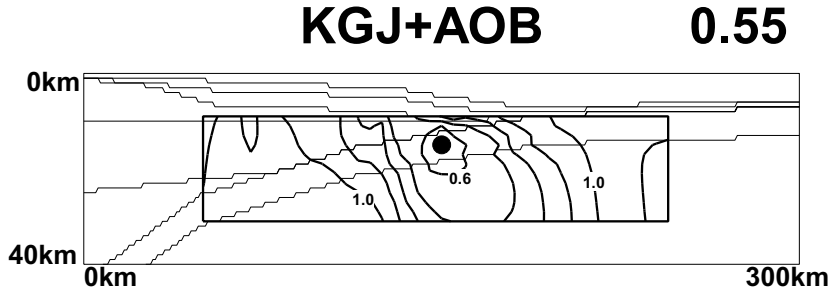

Fig. 7. The contour plot of the residuals projected on to the vertical cross section of the assumed structure. The contour interval is 0.1 . Solid circles indicate the optimum source points. The number attached to each cross section is the minimum value of the residuals. 


\section{KGJ+AOB}

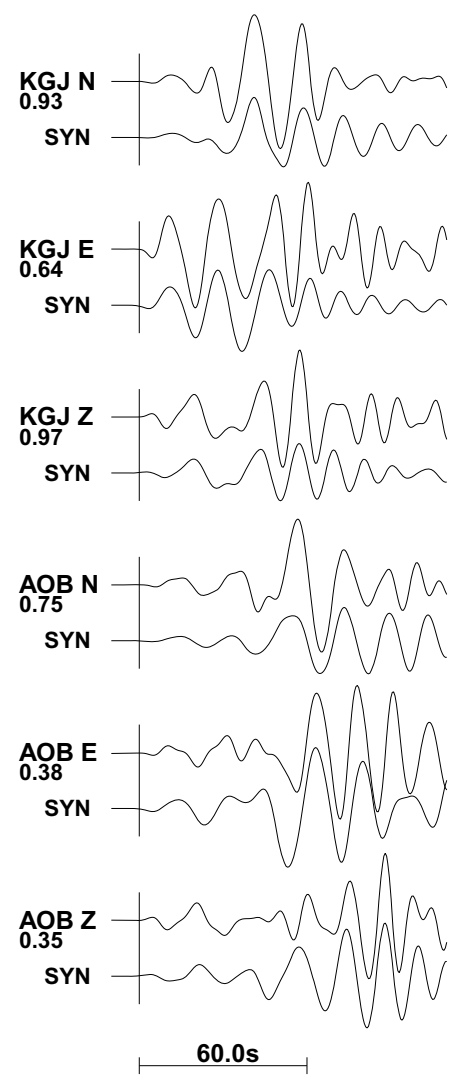

Fig. 8. The observed and synthetic waveforms calculated by the two-station inversion. The upper trace in each component is the observed one Vertical lines indicate the onsets of the $P$-waves. Maximum amplitudes in $\mathrm{mm}$ are also indicated.

vertical direction. The solution of the single-station inversion for AOB (middle) falls into a local minimum discussed above so that the position is not reasonable for this type of event. The result of the two-station inversion (bottom) indicate a well defined minimum at a reasonable position. Thus, as well as the source depth, the source location is well constrained both in transverse and radial directions in the twostation inversion.

Figure 8 shows the observed and the synthetic waveforms for the two-staion inversion. The dispersive feature in the surface wave is still clearly preserved and the first motions (i.e., polarity of the $P$-wave) of all the synthetics are consistent with the data.

\section{Discussion and Conclusion}

The focal depths of the events of the seismic activity in northern Japan trench have been precisely determined by land-side observations of the depth phases (i.e., $S$ to $P$ conversion at the surface: Umino et al., 1995) and by in-situ observations by the ocean bottom seismometers (Hino et al., 1996). These reports determined the focal depths of the events in the region studied in this paper to be around $15 \mathrm{~km}$ with a scatter of about $\pm 5 \mathrm{~km}$. The optimum depth obtained by our waveform inversion $(15 \mathrm{~km})$ is in good agreement with these precisely determined source depths.

Single-station inversion using the near-source seismic recordings is sometimes inevitable as the good near-source recordings is often rare in many regions (e.g., Delouis and Legrand, 1999). There is a similar situation in the subduction region: the station coverage is often one-sided and not quite good because of the presence of the ocean where seismic stations are very rare. Even under such situations, we have demonstrated that reasonable moment tensor and source location can be estimated by the waveform inversions with the reciprocal finite difference Green's function, provided that data from two (or more) stations are available.

We have also demonstrated the strong effect of the inhomogeneous structure (Fig. 4) that cannot be modeled by any flat-layered model. Such effects must be considered carefully, especially in the waveform study of the rupture process on the fault plane (e.g., Mori and Shimazaki, 1985; Nakayama and Takeo, 1997) as the location of the subevents are determined solely based on the waveform data.

The conclusions of this paper are: (1) The current knowledge on the subduction region structure is practically effective in reproducing its large effects on the observed waveforms. (2) An efficient "reciprocal" moment tensor inversion method is developed. (3) Reasonable moment tensors are obtained by the reciprocal inversion. The fit of the synthetics to the observed waveforms is also good. (4) The residual distributions indicate somewhat non-unique behavior in the estimation of the epicentral location especially for the singlestation inversions. The epicentral solution for the two-station inversion is more stable.

Acknowledgments. The author is grateful to the staffs in Research Center for Prediction of Earthquakes and Volcanic Eruptions, Graduate School of Science, Tohoku University and Earthquake Research Institute, University of Tokyo for their online data base. The author is also grateful to Yasuhiro Yoshida, Yasumaro Kakehi and Göran Ekström for critically reviewing the manuscript. Discussions with Naoki Kobayashi were also helpful. This research is partially supported by the Grant-in-Aid for Scientific Research (C) (No. 08640527) from the Ministry of Education, Science, Sports and Culture of Japan. One of the calculations was employed in Earthquake Research Institute, University of Tokyo.

\section{References}

Delouis, B. and D. Legrand, Focal mechanism determination and identification of the fault plane of earthquakes using only one or two near-source seismic recordings, Bull. Seism. Soc. Am., 89, 1558-1574, 1999.

Dziewonski, A. M. and J. H. Woodhouse, Studies of the seismic source using normal mode theory, Proc. Enrico Fermi Sch. Phys., 85, 45-137, 1983.

Fukuyama, E., M. Ishida, D. S. Dreger, and H. Kawai, Automated seismic moment tensor determination by using on-line broadband seismic waveforms, Zisin (J. Seism. Soc. Japan, 2nd series), 51, 149-156, 1998 (in Japanese with English abstract).

Furumura, T. and H. Takenaka, 2.5-D modelling of elastic waves using the pseudospectral method, Geophys. J. Int., 124, 820-832, 1996.

Hino, R., T. Kanazawa, and A. Hasegawa, Interplate seismic activity near the northern Japan Trench deduced from ocean bottom and land-based seismic observations, Phys. Earth Planet. Inter., 93, 37-52, 1996.

Kawakatsu, H., Automated near-realtime CMT inversion, Geophys. Res. Lett., 22, 2569-2572, 1995.

Kikuchi, M. and H. Kanamori, Inversion of complex body waves-III, Bull. Seism. Soc. Am., 81, 2335-2350, 1991.

Mori, J. and K. Shimazaki, Inversion of intermediate-period Rayleigh waves for source characteristics of the 1968 Tokachi-Oki earthquake, J. Geophys. Res., 90, 11374-11382, 1985.

Murauchi, S. and W. Ludwig, Crustal structure of the Japan trench: the effect of subduction of ocean crust, in Scientific Party, in Initial Reports of the Deep Sea Drilling Project, 56, 57, Pt. 1, U.S. Govt. Printing Office, 
Washington, 1980.

Nakayama, W. and M. Takeo, Slip history of the 1994 Sanriku-Haruka-Oki, Japan, earthquake deduced from strong-motion data, Bull. Seism. Soc. Am., 87, 918-931, 1997.

Okamoto, T., Location of shallow subduction zone earthquake inferred from teleseismic body waveform, Bull. Seism. Soc. Am., 84, 264-268, 1994a.

Okamoto, T., Teleseismic synthetics obtained from 3-D calculations in 2-D media, Geophys. J. Int., 118, 613-622, 1994b.

Pasyanos, M. E., D. S. Dreger, and B. Romanowicz, Toward Real-Time Estimation of Regional Moment Tensors, Bull. Seism. Soc. Am., 86, 1255-1269, 1996.

Sipkin, S. A., Rapid determination of global moment-tensor solutions, Geophys. Res. Lett., 21, 1667-1670, 1994.

Suyehiro, K., T. Kanazawa, and H. Shimamura, Air gun-ocean bottom seismograph seismic structure across the Japan trench area, in Initial
Reports of the Deep Sea Drilling Project, edited by H. Kagami, D. E. Karig, W. T. Coulbourn et al., 87, U.S. Govt. Printing Office, Washington, 1980.

Tanimoto, T., Modelling curved surface wave paths: membrane surface wave synthetics, Geophys. J. Int., 102, 89-100, 1990.

Thio, H. K. and H. Kanamori, Moment tensor inversions for local earthquakes using surface waves recorded at TERRAscope, Bull. Seism. Soc. Am., 85, 1021-1038, 1995.

Umino, N., A. Hasegawa, and T. Matsuzawa, $s P$ depth phase at small epicentral distances and estimated subducting plate boundary, Geophys. J. Int., 120, 356-366, 1995.

T. Okamoto (e-mail: taro@geo.titech.ac.jp) 